\section{THE UTILITY OF AI-POWERED SPATIAL CLASSIFICATION OF INTRATUMORAL CD8+ IMMUNE-CELL DISTRIBUTION IN PREDICTING OVERALL SURVIVAL IN PATIENTS WITH MELANOMA AS PART OF THE CHECKMATE 067 CLINICAL TRIAL}

George Lee*, Keyur Desai, Hao Tang, Daniel Cohen, Scott Ely, John Wojcik, Jimena TrilloTinoco, Benjamin Chen, Akshita Gupta, Daniel Tenney, Vipul Baxi, Robin Edwards, Megan Wind-Rotolo. Bristol Myers Squibb, Princeton, NJ, USA

Background Spatial patterns of CD8 $+\mathrm{T}$ cells in the tumor microenvironment are associated with clinical outcomes in patients with advanced solid tumors. However, attempts to quantify spatial topology are hindered by challenges in manual scoring, heterogeneous immune-cell infiltrates, and interpathologist variability. Artificial intelligence (AI)-powered analysis can quantify CD8 topology in a biologically meaningful, reproducible, and scalable way. Using an AI-driven algorithm, we retrospectively assessed CD8 topology as a biomarker of response to immunotherapy in patients with advanced melanoma.

Methods We trained a random forest classifier to predict CD8 topology using parenchymal and stromal CD8 + immune-cell measurements derived from a deep-learning platform (PathAI, Boston, MA). For model validation, pathologists manually classified CD8 immunohistochemistry (C8/144B, Agilent, Santa Clara, CA) in melanoma samples into inflamed (CD8+ cells in tumor parenchyma), excluded $(\mathrm{CD} 8+$ cells restricted to stroma), and desert (deficient in $\mathrm{CD} 8+$ cells) patterns. We explored the association with overall survival (OS) in a subset of patients with previously untreated metastatic melanoma who received nivolumab + ipilimumab (NIVO+IPI, $n=102)$ or NIVO alone $(n=107)$ in the CheckMate 067 phase 3 trial. Retrospective analysis of baseline AI-defined CD8 topology was performed alone and combined with manually scored programmed death ligand 1 (PD-L1) expression on tumor cells.

Results Classifier model predictions were concordant with manual scoring (determined by a consensus of pathologists) and non-inferior to the agreement between 2 pathologists, via Cohen's kappa coefficient $\mathrm{k}=0.79$ and $\mathrm{k}=0.65$, respectively. No statistically meaningful differences in outcomes were observed between CD8-excluded and CD8-inflamed phenotypes within the PD-L1 $\geq 1 \%$ population. However, patients with PD-L1 <1\%/CD8-excluded tumors exhibited longer median OS compared with those with PD-L1 <1\%/CD8inflamed (table 1). $38 \%(40 / 104)$ of PD-L1 < $1 \%$ tumors were CD8-excluded. Within PD-L1 <1\%, patients with an excluded phenotype also exhibited lower frequency of severe adverse events (grade $\geq 3$ ) than patients with inflamed phenotype following treatment: NIVO+IPI, 75\% $(n=20)$ vs 91\% ( $=11)$; NIVO, 61\% $(n=18)$ vs $80 \%(n=15)$. Compared with PD-L1 status, the composite biomarker (AI-classified CD8-excluded plus PD-L1 $\geq 1 \%$ ) identified a larger group of patients who had greater survival benefit with NIVO+IPI or NIVO alone (table 2).
Abstract 387 Table 1 Immunotherapy outcomes by CD8+ topology in PD-L1<1\% melanoma

\begin{tabular}{|l|c|c|c|c|}
\hline Treatment arm & \multicolumn{2}{|c|}{ NIVO+IPI } & \multicolumn{2}{c|}{ NIVO } \\
\hline Phenotype (n) & $\begin{array}{c}\text { PD-L1 <1\%, CD8- } \\
\text { excluded (20) }\end{array}$ & $\begin{array}{c}\text { PD-L1 <1\%, CD8- } \\
\text { inflamed (12) }\end{array}$ & $\begin{array}{c}\text { PD-L1 <1\%, CD8- } \\
\text { excluded (20) }\end{array}$ & $\begin{array}{c}\text { PD-L1 <1\%, CD8- } \\
\text { inflamed (15) }\end{array}$ \\
\hline $\begin{array}{l}\text { Median OS } \\
\text { (months) }\end{array}$ & $>50$ & 10.1 & $>50$ & 25.8 \\
\hline OS HR (95\% Cl) & \multicolumn{2}{|c|}{$0.23(0.09-0.61), P<0.01$} & \multicolumn{2}{|c|}{$0.68(0.27-1.70), P=0.41$} \\
\hline
\end{tabular}

In a subset of patients with melanoma and tumor cell PD-L1 expression $<1 \%$ in the CheckMate 067 clinical trial, those with a CD8-excluded phenotype demonstrated longer overall survival compared with those with a CD8-inflamed phenotype when treated with NIVO \pm IPI.

\begin{tabular}{|c|c|c|c|c|}
\hline \multirow{2}{*}{$\begin{array}{l}\text { Treatment arm } \\
\text { (n) }\end{array}$} & \multicolumn{2}{|c|}{ PD-L1 $\geq 1 \%$} & \multicolumn{2}{|c|}{$\begin{array}{c}\text { Composite biomarker } \\
\text { (PD-L1 } \geq 1 \% \text { plus CD8-excluded) }\end{array}$} \\
\hline & $n(\%)$ & OS HR $(95 \% \mathrm{Cl})$ & $n(\%)$ & OS HR $(95 \% \mathrm{Cl})$ \\
\hline NIVO+IPI (102) & $52(51 \%)$ & $\begin{array}{c}0.50(0.29-0.89), \\
P=0.017\end{array}$ & $72(71 \%)$ & $\begin{array}{c}0.35(0.20-0.61) \\
P<0.001\end{array}$ \\
\hline NIVO (107) & $53(50 \%)$ & $\begin{array}{c}0.46(0.27-0.79), \\
P=0.005\end{array}$ & $73(68 \%)$ & $\begin{array}{c}0.37(0.22-0.62) \\
P<0.001\end{array}$ \\
\hline
\end{tabular}

In patients with melanoma in the CheckMate 067 clinical trial, the composite biomarker (AI-classified CD8-excluded phenotype plus PD-L1 expression $\geq 1 \%$ ) identified more biomarker-positive patients and demonstrated increased overall survival benefit vs PD-L1 status alone for patients treated with $\mathrm{NIVO} \pm$ IPI. Hazard ratios represent patients with a PD-L1 expression of $\geq 1 \%$ compared with PD-L1 $<1 \%$ or patients with a PD-L1 expression of $\geq 1 \%$ and CD8-excluded phenotype compared with PD-L1 expression $<1 \%$ and not CD8excluded.

Conclusions This study explores the utility of combining AIpowered CD8 topology classifications with PD-L1 expression as a composite biomarker associated with immunotherapy response. In patients with $\mathrm{PD}-\mathrm{L} 1<1 \%$ melanoma, median OS with NIVO+IPI was significantly longer in patients with CD8excluded tumors than with an inflamed phenotype. Further studies are underway to identify mechanisms underlying responses to NIVO+IPI.

Acknowledgements We would like to thank the team at PathAI for development of the AI classifier, and Dako, an Agilent Technologies, Inc. company, for collaborative development of the PD-L1 IHC 28-8 pharmDx assay. Editorial support was provided by Emily Motola, PharmD, and Matthew Weddig of Spark Medica Inc.

Trial Registration ClinicalTrials.gov number NCT01844505

Ethics Approval The study protocol and all amendments were approved by local institutional review boards, and the protocol was conducted in accordance with the Declaration of Helsinki and Good Clinical Practice Guidelines, as defined by the International Conference on Harmonisation of Technical Requirements for Pharmaceuticals for Human Use. All patients provided written informed consent before enrollment.

http://dx.doi.org/10.1136/jitc-2021-SITC2021.387 